\title{
Evaluation of socio-economic impacts of the business R\&D support in small economies. The case of the Czech Republic
}

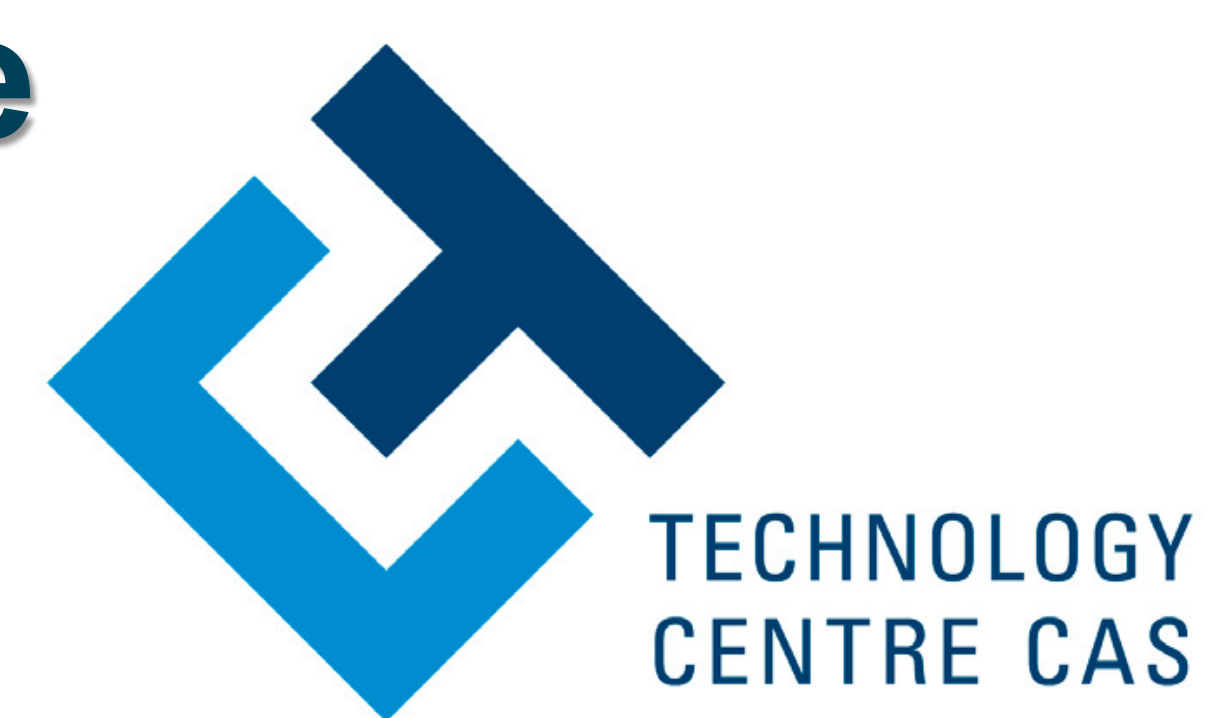

Tomas Ratinger, Vladislav Cadil, Sylvester Amoako Agyemang

Technology Centre CAS

\begin{abstract}
Context
The counter-factual econometric analysis is becoming increasingly popular particularly for the assessment of input and output additionality. The use of methods based on comparison between strictly separated treated and control groups faces some obstacles in terms of data-availability and quality, interpretation and presentation of results to a non-specialist audience. A particular challenge is finding the right control group, particularly if country or investigated region is small and support
measures plentiful. If an appropriate control group cannot be found among the unsupported companies, a so-called generalized propensity score matching (GPSM) can be used, provided that firms receive a different level of treatment (support). The control group is created from the closest similar firms, which received a different level of support.

Research aims

$>$ To assess the effects of the Czech programme TIP supporting R\&D in private companies in the recent decade.

To point out the methodological challenges of such an assessment, and to show how they can be addressed using the GPSM approach.
\end{abstract}

\section{Data}

Two data sources were used: monitoring data of the support programme TIP and economic data from the database Bisnode-MagnusWeb.

The impact is measured at two hierarchical levels - the firm (micro) and the economy (macro) levels. At the micro level, the impact of R\&D support on the firms' performance are measured by 5 indicators: 2 measuring net and gross R\&D expenditure effects and 3 indicators relating to output - gross value added, profit and labour productivity. The macro level impact of R\&D support is represented only by a fiscal indicator - average tax paid by the firms.

The assessment of the R\&D expenditure effects relates to the period of 2010 to 2016 while the impact of the support on the output indicators relates to 2013-2015 (the period after the economic recession 2009-2012). In the both cases, the year of 2009 was used as the base year for the analyses. For each of the output indicator, the difference-in-difference approach is adopted in order to remove the time invariant "fixed effects" $\rightarrow$ the difference between the average figure for the "impact period" (2013 to 2015) and the base year (2009) is computed.

\section{Methodology}

Size categories of the beneficiary firms

The probability of receiving a lower or higher support and treatments effects are more likely to be heterogeneous with respect to the firm size. Therefore the firms are divided into three categories (small, medium and large) using their annual turnover in the base year.

Control Variables

To deal with the pre-treatment biases in the impact estimation, 10 pre-treatment covariates (cash flow, fixed assets, current assets, equity, profit, long term debt/total assets, bank credit/total assets, intermediate consumption, depreciation, interest received by a firm) referring to the base year (2009) are selected and included in the model.

A Linear Regression Analyses of the Outcome and Control Variables

Firstly, the impact of R\&D support on the defined outcome/performance variables is analysed by regressing the control variables and the treatment variable (R\&D support) on the outcome variables with the aim to determine the relevance of the selection of the control (pre-treatment) variables in respect of the formation of the performance variables.

\section{The Generalised Propensity Score (GPS) Matching}

Afterwards, the conditional distribution of the treatment (support) given the covariates via Ordinary Least Squares (OLS) method is estimated. Then the normality of the disturbances using the Kolmogorov-Smirnov equality-of-distributions test is tested. $\rightarrow$ GPS estimation

The next step is to test whether the propensity scores balance the sample. To achieve this, the treatment (support) is divided into 3 different intervals in our particular case.

Estimation of the Dose-response Function

Finally, each outcome variable (in all size categories of firms) is regressed on the conditiona expected value of R\&D support given by the pre-treatment variables $\rightarrow$ dose-response functions. The Dose-Response Function indicates the estimated impact of a given level of R\&D support on the outcome indicator. In the way answering the counterfactual question, what would have happened to a given firm had that unit received a different level of treatment.

(Marginal) Treatment Effect Function shows the change of the outcome indicators in response to the change of the treatment level.

Results (4 of 7 indicators)

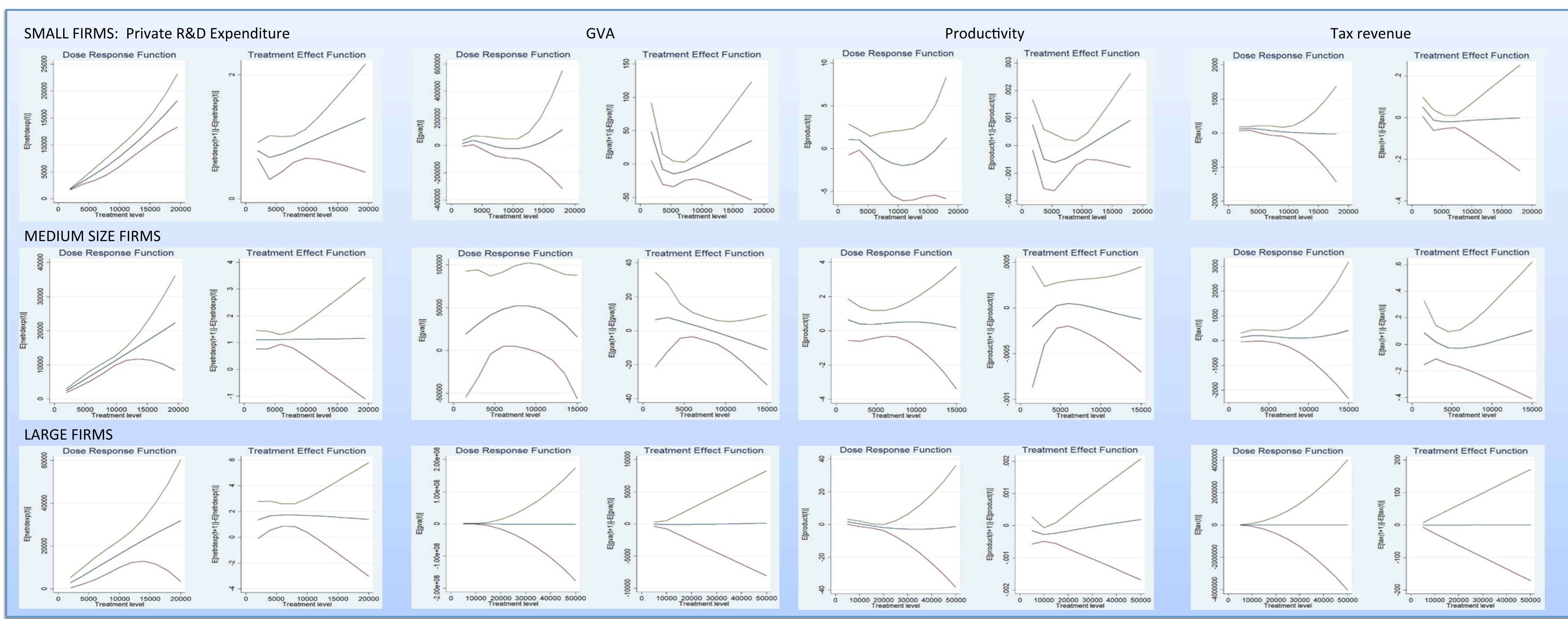

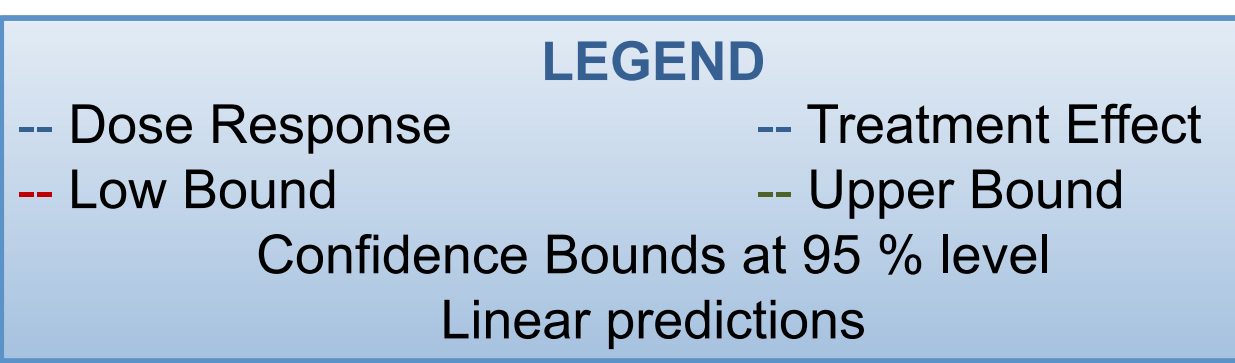
Linear predictions

\section{Conclusions}

Treatment effects are heterogeneous depending on firm sizes. Generally, the R\&D support effects are positive and more substantial in small and medium scale firms while there are no such effects confirmed for large-scale firms.

> The support could only impact positively on the output (profit, productivity and GVA) of small-scale firms only after reaching a certain minimum threshold of the support whereas output diminishes for medium scale firms after a point of saturation.

> The support has a complementary effect rather than a substitutability effect on the private R\&D expenditures.

The differences in impacts between the size categories of supported enterprises can be explained by the different economic performance of small The differences in impacts between the size categories of supported enterprises can be explained by the different economic performance of small
firms compared to the medium and large companies and different strategic focus of R\&D in each size category. Small companies are more likely to concentrate on activities with faster return on capital. Therefore R\&D conducted by small firms is rather oriented on the direct support of their production activities or on the application of the new knowledge in the short-term horizon, while the strategic focus of R\&D in large companies are more focused on long-term strategic research.

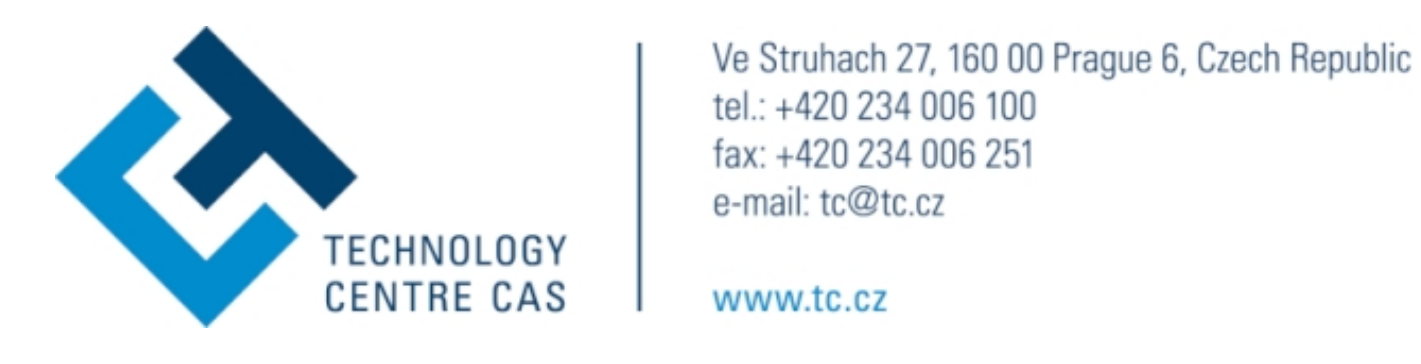

